\title{
Catheter ablation of synchronous left fascicular ventricular tachycardia and atrioventricular node reentry tachycardia
}

\author{
Wei Zhang ${ }^{1}$, Shijie $\mathrm{Li}^{2}$, Guangxin Wang ${ }^{1}$, Xianjin $\mathrm{Li}^{3}$, and Bing $\mathrm{Han}^{3}$ \\ ${ }^{1}$ Jinan Central Hospital Affiliated to Shandong University \\ 2 the First Affiliated Hospital of Nanjing Medical University \\ ${ }^{3}$ The Central Hospital of Xuzhou City
}

June 3,2020

\begin{abstract}
A 26-year-old man suffered from recurrent palpitation and his 12-lead electrocardiogram documented the alternating narrow and wide complex tachycardia. Electrophysiologic study revealed the synchronous left fascicular ventricular tachycardia (LFVT) and slow-slow atrioventricular node reentry tachycardia (ss AVNRT), and both of them were ablated successfully.
\end{abstract}

\section{Case report}

A 26-year old man underwent an electrophysiologic (EP) study and catheter ablation because of recurrent palpitation. 12-lead surface electrocardiogram (ECG) documented the alternating narrow and wide complex tachycardia. The ECG during the sinus rhythm and the echocardiography were normal.

The operation was performed under local anesthesia. Multipolar electrode catheters were placed to the coronary sinus, His bundle region and right ventricular apex of the patient respectively. The intracardiac electrogram during sinus rhythm was recorded, with the HV interval of $52 \mathrm{~ms}$. Wide complex tachycardia (cycle length $358 \mathrm{~ms}$ ) was induced by ventricular $\mathrm{S}_{1} \mathrm{~S}_{2}$ stimulation. The QRS complex morphology presented as right bundle branch block pattern (rSR') in lead V1. The rate of the ventricle was higher than that of the atrium. The His bundle potential preceded ventricular wave with a shorter HV interval (16ms) than that (52ms) during sinus rhythm (Figure1.A). Atrial burst pacing at a cycle length of $300 \mathrm{~ms}$ had no effect on QRS morphology and R-R interval. Based on these findings, the wide QRS complex tachycardia could be identified as LFVT.

During the onset of tachycardia, the atrial rate speeded up and the V-A relationship changed to 1:1, the morphology of the QRS complex changed between narrow and fused, with inconstant AV and VA interval. (Figure 1.B).Besides that, the wide complex tachycardia could occur immediately after the termination of the narrow complex tachycardia. The HV interval $(52 \mathrm{~ms})$ during narrow complex tachycardia was identical to that during the sinus rhythm (Figure 1.C). The surface electrocardiogram showed that the morphology of the QRS complex alternately transformed among wide, narrow and fused. The cycle length of the tachycardia fluctuated between $341 \mathrm{~ms}$ and $358 \mathrm{~ms}$ (Figure 1.D). Based on these characteristics, the supraventricular tachycardia (SVT) was ascertained.

LFVT was induced repeatedly by spontaneous premature ventricular beat and ablated primarily. An irrigated Thermocool Navistar catheter (Biosense Webster, Inc) was advanced in the left ventricle via the right femoral artery. Ablation was applied at the site of the earliest Purkinje potential during tachycardia and rendered LFVT non-inducible. 
After that, atrial S1S2 stimulation revealed AV conduction jumping phenomenon. The narrow complex tachycardia was still easily induced by ventricular stimulation. During tachycardia, The RP' inter$\operatorname{val}(191 \mathrm{~ms})$ was longer than P'R interval (159ms) (Figure2.A). When ventricular pacing (SS=330ms) was delivered at a rate faster than the tachycardia, the ventricle was captured without changing the cycle length of the AA interval (345ms), which ruled out atrioventricular reentrant tachycardia. The earliest retrograde atrial activation recorded at the ostium of the coronary sinus was same to that recorded during the tachycardia, which implied the unlikely of atrial tachycardia. (Figure2.B). It was also found that the tachycardia could be terminated by ventricular stimulation $(\mathrm{SS}=320 \mathrm{~ms})$ without retrograde ventricular-atrial conduction and so atrial tachycardia was excluded (Figure2.C) [1]. Based on the above findings, slow-slow AVNRT was diagnosed. Successful ablation was achieved near the CS ostium guided by the fluoroscopy during tachycardia. Repeated EP study showed that there was neither retrograde VA conduction during ventricular pacing (Figure2.D) nor AV conduction jumping phenomenon during atrial extra-stimulation. During one-year follow-up, no recurrence was found.

\section{Discussion}

We reported an anecdotal case of pararrhythmia with LFVT and ss AVNRT. Co-existence of LPFVT and AVNRT in one patient is rare. They can occur spontaneously at different times or be induced separately by atrial or ventricular stimulation [2]. Extremely limited literatures reported simultaneous LPFVT and slow-fast AVNRT after intravenous administration of atropine [3]. The mechanism of the pararrhythmia was attributed to two coexisting reentrant circuits with similar cycle lengths by chance. During the procedure, it could be seen that the QRS complex morphology alternately transformed among wide, narrow and fusive, accompanied by change of HV interval that shortened or lengthened following QRS complex widened or narrowed. This phenomenon indicated a concurrent attack of two tachycardias, which should be distinguished from supraventricular tachycardia with intermittent bundle branch block. In the latter case, there shouldn't be obvious shortening of HV interval. Considering that both LPFVT and AVNRT were originated from the $\mathrm{AV}$ conduction system, maybe there is an unconfirmed interaction between the two kinds of arrhythmias.

\section{Conclusion}

An extremely rare pararrhythmia with LFVT and ss AVNRT was reported. It implied that two independent but possibly interplayed reentrant circuits located at the AV node and the left fascicle simultaneously.

\section{Reference}

[1].Yukoh Hirai, Ming-Shien Wen, San-Jou Yeh, et al. A Specific Sign for Differential Diagnosis of Atypical Atrioventricular Nodal Reentrant Tachycardia from Atrial Tachycardia. Pacing Clin Electrophysiol[J]. 2012;35(2):245-8.

[2].Fa-Po Chung, Vu Van Ba, Yenn-Jiang Lin, et al. The Prevalence and Characteristics of Co-existed Atrioventricular Nodal Reentrant Tachycardia and Idiopathic Left Fascicular Ventricular Tachycardia. Journal of Cardiovascular Electrophysiology[J].2018;29(8):doi: 10.1111/jce.13628.

[3]. Belhassen B, Pelleg A, Paredes, et al. Simultaneous AV nodal reentrant and ventricular tachycardias. Pacing Clin Electrophysiology[J]. 1984;7(3):325-31. 


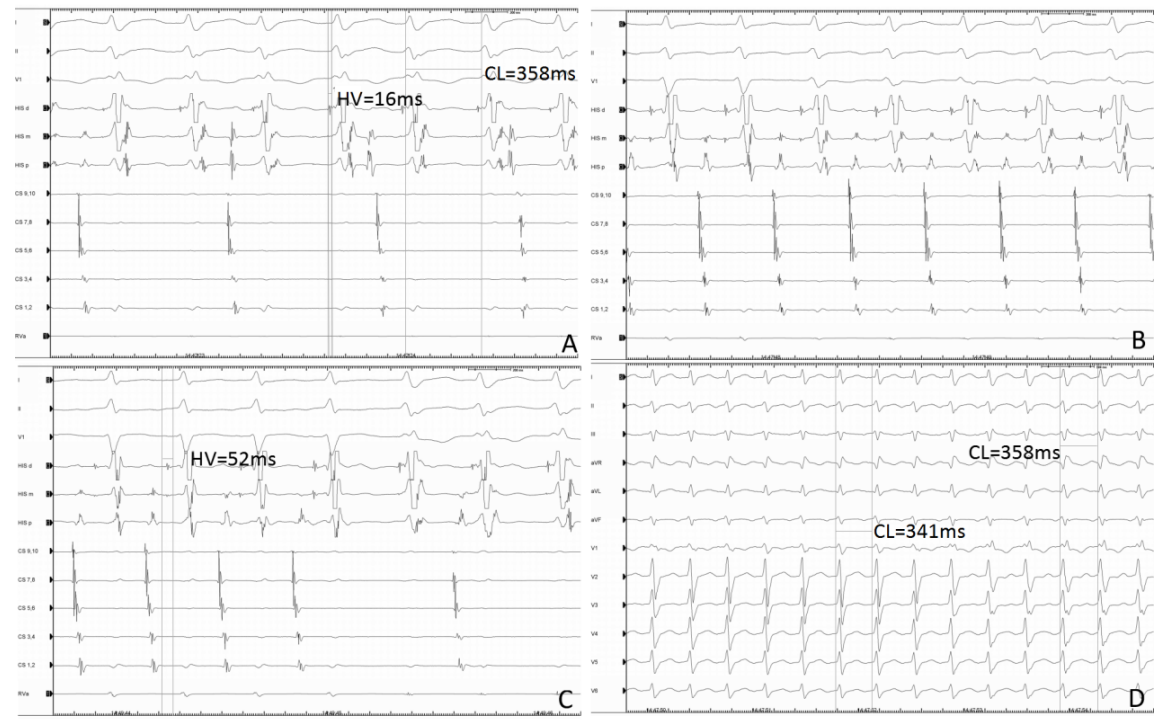

Figure1. A. Wide complex tachycardia (cycle length $358 \mathrm{~ms}$ ) presented as right bundle branch block pattern (rSR') in lead V1. The rate of the ventricle was higher than that of the atrium. The His bundle potential preceded ventricular wave with a shorter HV interval (16ms) than that (52ms) during sinus rhythm. B. During the onset of tachycardia, the atrial rate speeded up and the V-A relationship changed to 1:1, the morphology of the QRS complex changed between narrow and fused, with inconstant AV and VA interval. C. The wide complex tachycardia could occur immediately after the termination of the narrow complex tachycardia. The HV interval (52ms) during narrow complex tachycardia was identical to that during the sinus rhythm. D. The surface electrocardiogram showed that the morphology of the QRS complex alternately transformed among wide, narrow and fused. The cycle length of the tachycardia fluctuated between $341 \mathrm{~ms}$ and $358 \mathrm{~ms}$. 


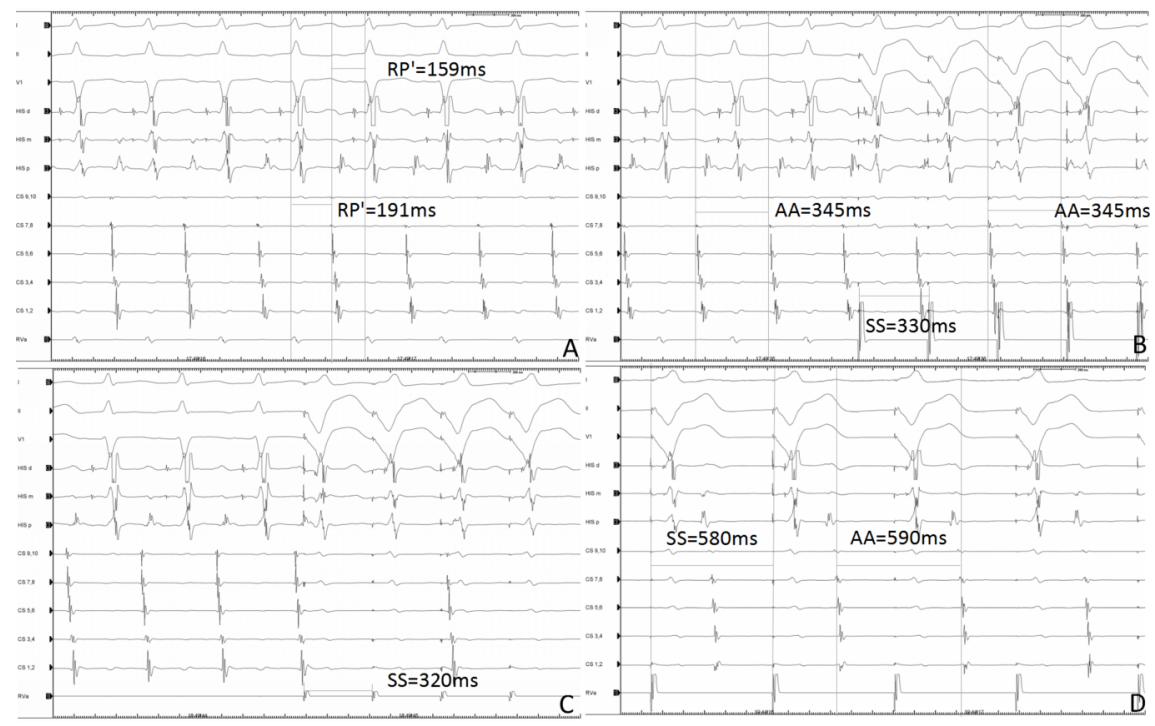

Figure 2. A. During the narrow complex tachycardia, The RP' interval(191ms)was longer than P'R interval $(159 \mathrm{~ms})$. B. When ventricular pacing $(\mathrm{SS}=330 \mathrm{~ms})$ was delivered at a rate faster than the tachycardia, the ventricle was captured without changing the cycle length of the AA interval (345ms), which ruled out atrioventricular reentrant tachycardia. The earliest retrograde atrial activation recorded at the ostium of the coronary sinus was same to that recorded during the tachycardia, which implied the unlikely of atrial tachycardia. C. The tachycardia could be terminated by ventricular stimulation (SS=320ms) without retrograde ventricular-atrial conduction and so atrial tachycardia was excluded. D. After successful ablation near the CS ostium, no retrograde VA conduction was observed during ventricular stimulation (580ms). 